\title{
The Effect of Plasma Density and Emitter Geometry on Space Charge Limits for Field Emitter Array Electron Charge Emission into a Space Plasma
}

\author{
Dave Morris $^{1}$, Brian Gilchrist ${ }^{1}$, Alec Gallimore ${ }^{2}$ \\ ${ }^{1}$ Space Physics Research Lab., U. of Michigan, Ann Arbor, MI 48109 \\ ${ }^{2}$ Plasmadynamics and Electric Propulsion Lab., U. of Michigan, Ann Arbor, MI 48109 \\ 734-763-5357; thecat@umich.edu \\ 734-763-6230; Brian.Gilchrist@umich.edu \\ 734-764-8224; rasta@umich.edu
}

\begin{abstract}
Field Emitter Array Cathodes (FEACs) are a new technology being developed for several potential spacecraft electron emission and charge control applications. Instead of a single hot (i.e. high powered) emitter, or a gas dependant plasma contactor, FEAC systems consist of many (hundreds or thousands) of small (micron level) cathode/gate pairs printed on a semiconductor wafer that effect cold field emission at relatively low voltages. Each individual cathode emits only micro-amp level currents, but a functional array is capable of amp/cm $\mathrm{cm}^{2}$ current densities. It is hoped that thus FEAC offers the possibility of a relatively low-power, simple to integrate, and inexpensive technique for the high level of current emissions that are required for an electrodynamic tether (EDT) propulsion mission. Space charge limits are a significant concern for the EDT application. Vacuum chamber tests and PIC simulations are being performed at the University of Michigan Plasmadynamics and Electric Propulsion Laboratory and Space Physics Research Laboratory to determine the effect of plasma density and emitter geometry on space charge limitations. The results of this work and conclusions to date of how to best mitigate space charge limits will be presented.
\end{abstract}

\section{INTRODUCTION}

Space electrodynamic (ED) tethers offer the opportunity for in-space "propellantless" propulsion and power generation around planets with a magnetic field and an ionosphere (e.g., Earth and Jupiter). In general, moving a conductor across a magnetic field generates an electromotive force (EMF) to drive current through the conductor if a means to "close the circuit" is available. For example, using gravity gradient stabilized space tethers around Earth, it is possible to have kilometer-scale structures that move across the geomagnetic field at rapid velocities generating 50-250 V/km EMF in an eastward-moving system at a mid- to low-latitude orbit inclination. Current flow through the tether is enabled by collecting electrons from the ionosphere at or along one end of the tether and, at the opposite end, either injecting electrons back into the ionosphere or collecting ions. Electron injection is necessary to achieve the highest possible currents given the low mobility of ions.

The focus of this paper is electron injection via FEAC systems. FEAC systems emit charge with an initial velocity depending on the gate-tip voltage, and the voltage of any other associated acceleration or protection grids. As charge leaves the cathode into free space, or a plasma, each charge emitted experiences a field created by the charges before it. If the emission density is too high, this effect will decelerate the charge and, in the limit, reverse the flow and reflect current back on the emitter (so called space charge limited emission (Luginsland, 1998)).

This space charge limitation is an important consideration for FEAC and other charge emission systems. The implications of this limitation and techniques for mitigating it are being studied at the University of Michigan 
and elsewhere (Marrese, 1999). The approach and results to date of these studies will be presented below. This paper will also cover some of the other issues facing FEAC systems in EDT propulsion.

\section{FEAC SYSTEMS}

The figure below shows a typical FEAC system. The scale is sub micrometer. A typical array is a semiconductor wafer with up to millions of individual tips per square centimeter. Typical operating voltages are in the range of 50-100 V, and typical currents can be on the order of $1 \mathrm{~mA}$ per tip.

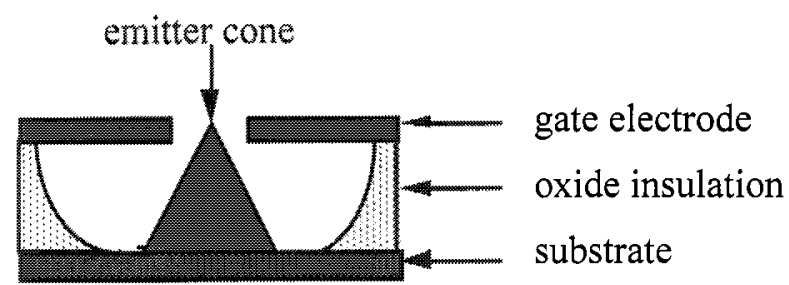

FIGURE 1. Schematic View of a Single Tip/Gate Structure. A typical FEAC will consist of thousandsmillions.

The expected advantages of FEAC systems over alternative electron emission devices are no consumables and moderate power requirements provided robust operation can be demonstrated in a non-ultra high vacuum spacecraft environment. Hollow cathode contactors, which operate by generating a dense plasma plume and injecting electrons into the ambient plasma across a double layer sheath, uses a consumable gas supply and requires heater power, but may need the least drive power to inject electrons into the plasma. Electron guns require higher injection voltages and power than FEACs, and are sensitive to pressure gradients.

\section{FEAC Electrical Configuration}

For a downward thrust (de-orbit) mission, energy for electron emission can come directly from the tether kinetic energy. For upward thrust it must come from solar cells or other onboard energy sources. In both cases decisions must be made as to how to connect the FEAC (gate and tips) to the spacecraft. How the gate and tip are biased relative to the spacecraft result in various advantages and disadvantages to the system overall.

A factor in this decision is that the spacecraft potential may vary widely. For standard west-to-east orbits, the bottom of the tether will be biased by the tether's motion through the earth's magnetic field possibly as many as hundreds or thousands of volts negative. As the FEAC operates, however, it can bring the spacecraft up to neutral bias and even above, if the FEAC's current provision capability exceeds the tether/upper spacecraft's current collection capability. Therefore care must be exercised in how the tip is biased relative to the gate and spacecraft.

The figures below illustrate three basic configurations. Advantages of grounded tip include stability and low return current through the gate-tip power supply. One disadvantage is that the emitted electrons must move away from a gate biased positive relative to the plasma (assuming the spacecraft is close to the plasma potential) and this will act as a decelerating force, increasing space charge limitations. 


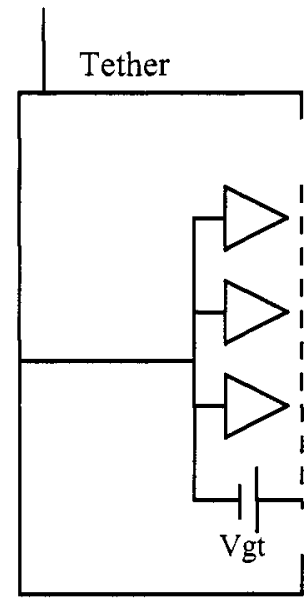

Basic Grounded Tip Configuration

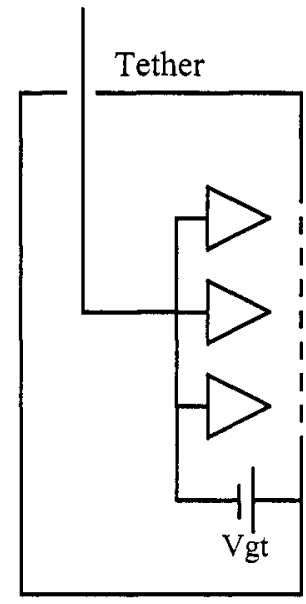

Basic Grounded Gate Configuration

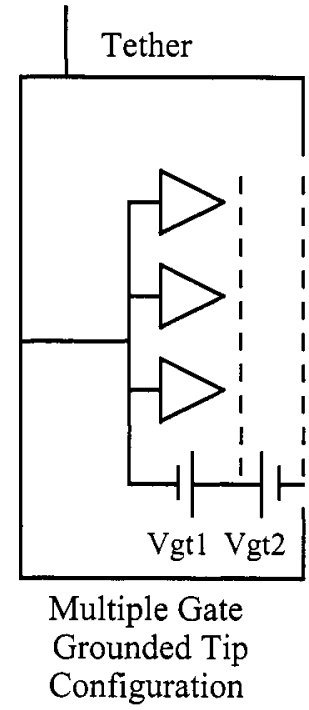

Multiple Gate

Grounded Tip

FIGURE 2. Three Possible Electrical Interfaces between a FEAC and a Spacecraft. Each has it's own advantages and disadvantages.

The advantage of grounded gate is that electrons leave through a screen at the same potential as the spacecraft, no decelerating fields will exist (or if the spacecraft is biased negative, a beneficial accelerating field will be present). One major disadvantage is that the gate-tip voltage can be directly effected by swings in the tether voltage. This becomes an especially significant concern when the tether is connected and disconnected for mission propulsion reasons- potentially resulting in several hundred volt spikes that the gate tip supply must suppress to avoid sparking the entire array.

Combinations of these techniques are possible as shown in the multiple gate grounded tip configuration. One advantage of a multiple gate configuration is that the second gate could be shaped to defocus the emitted electron beam, further mitigating space charge effects by reducing the effective density (see space charge limits below $).($ Vgt $=$ gate tip voltage $)$

\section{SPACE CHARGE LIMITS}

Charge control can be described as two major steps- 1) inject charge, 2) get the charge away. FEAC systems inject charge with low power and voltage, thanks to the small scale of the tip-gate geometry. But regardless of the voltage required to pull electrons free of the tips, they must leave the FEAC with enough energy to get away and stay away from the spacecraft. This is where space charge limitations come in.

The first region of concern for space charge reasons is in the sheath region, the plasma depleted area immediately surrounding the spacecraft. Plasma accommodation is of little assistance here, while the emitted electron density is the highest. It is the region where the electron velocity is the highest, and barring large spacecraft biases, the sheath will only be on the order of centimeters wide. Next the electrons must be accommodated by the plasma without significant reflection. Basically the electrons must spread out over sufficient distance such that the emitted electron density is less than the background density.

\section{D Space Charge Limits}

To illustrate issues with space charge limitations the following figures show results of one dimensional PIC simulation in the space charge limited regime. XPDP1 (Verboncoeur et al., 1993) simulates particle activity between two plates- with each particle representing a sheet of charge (thus the 1D nature). The plot on the left below is of velocity versus position of the individual electrons in the simulation (the apparently continuous line 
is actually individual electrons in close proximity). The center of the vertical axis is zero velocity. Electrons are emitted with $60 \mathrm{eV}$, but the space charge effect decelerates and actually reverses the direction of some. It should be noted that the figure below is not a steady state solution but rather a continually shifting distribution. (A snapshot is shown.) The figure on the right shows the time variation of the emitted current.
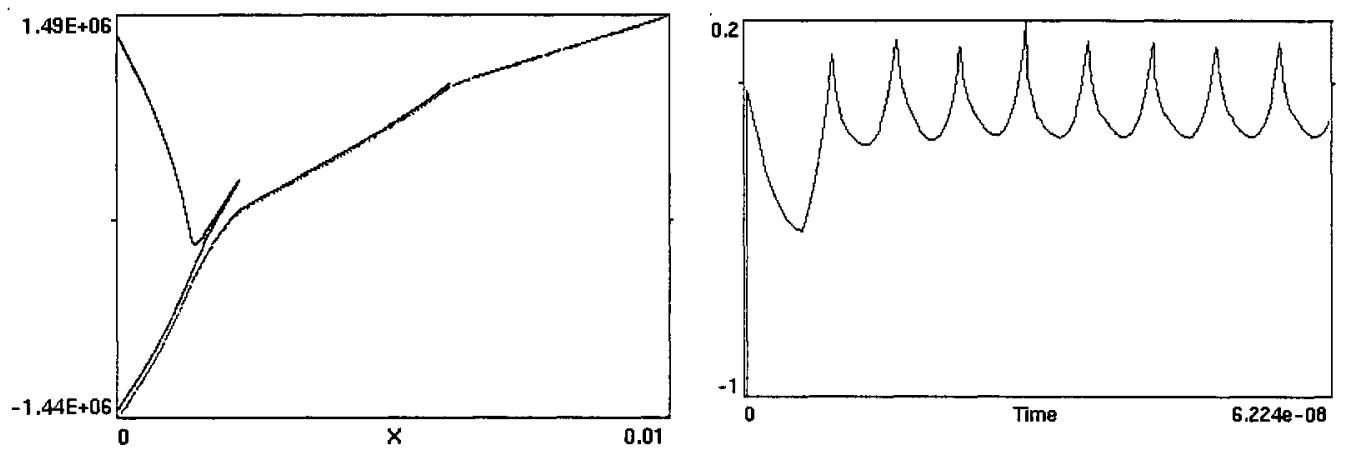

FIGURE 3. 1 Dimensional Charge Emission as Electron Velocity versus Position, and the Corresponding Current versus Time, both in the Space Charge Limited Regime.

This simulation represents the approximation where the sheath is considered to be the only region of interesti.e. that any electrons managing to cross the sheath are absorbed by a virtual anode (the ionosphere) and need no longer be considered. The simulation uses $1 \mathrm{~A}$ current emission across a $1 \mathrm{~cm} 1 \mathrm{~V}$ sheath. This simplest version of the problem has been solved analytically. The maximum current density that can be transmitted across a onedimensional planar gap is given by a one dimensional Child-Langmuir Law (Luginsland, 1998) that allows for non-zero emission velocity.

$$
I_{C L}(1)=\frac{4 \varepsilon_{0}}{9 e} \sqrt{\frac{2}{m_{e}}} \frac{T_{0}^{3 / 2}}{D^{2}}\left[1+\sqrt{1+\frac{e V}{T_{o}}}\right]^{3} A
$$

The A term has been added to describe the area of the emitter. This equation is used as a baseline reference in chamber simulations.

\section{Geometric Spreading}

Space charge limits are a function of emitted charge density. Therefore the more the emission beam is spread, the less the space charge limitations. Higher spatial dimensions allow for larger current emission. The following equation shows the increase in space charge limited current for two dimensions as opposed to one (Luginsland, 1996):

$$
\frac{J_{C L}(2)}{J_{C L}(1)}=1+\frac{0.3145}{W / D}-\frac{0.0004}{(W / D)^{2}}
$$

An additional improvement is allowed by the transition to three dimensions, as calculated by (Humphries, 1990):

$$
\frac{J_{C L}(3)}{J_{C L}(1)}=\frac{\left[r_{b}^{2}+(D / 2)^{2}\right]}{r_{b}^{2}}=\left[1+\left(D / 2 r_{b}\right)^{2}\right]
$$




\section{PIC Simulations}

The basic analysis is done in 1 dimensional pic code. In addition, confirmations of the above higher dimensionality equations as they apply to this application are done with XOOPIC (2dimensional PIC) and other codes in 2, 2.5, and 3 dimensions.

To quantify parameters of electron charge emission into a space plasma, a typical EDT propulsion mission operates in the low earth orbit environment, through the $\mathrm{F}$ and possibly as low as $\mathrm{E}$ regions of the ionosphere. From $100-1000 \mathrm{~km}$, ionosphere electron densities are typically within the outer extremes of $\mathrm{N}=10^{8}$ to $10^{13} \mathrm{~m}^{-3}$. (Tascione, 1988) For useful propulsion, tether currents (and therefore system emission currents) on the order of 1-3A are required at a minimum, with larger currents desirable. With limits in available spacecraft surface area, this implies an emission current on the order of $100 \mathrm{~mA} / \mathrm{cm}^{2}$. In order to minimize the power drain of the system, either from internal power, or from the tether emf used for propulsion, it is desirable to minimize the emission velocities. Initial estimates indicate electron emission energies on the order of tens of volts, in the 30-60 V range.

With a tethered satellite system, the bias of the spacecraft with respect to the plasma could be quite large- on the order of tens of volts or higher depending on the efficiency of charge emission. Bias voltages ranging from about half a volt to tens of volts can be considered. The Debye length in a plasma with a representative electron temperature of $0.1 \mathrm{eV}(\mathrm{NRL}, 1998)$ calculates out to $.7 \mathrm{~mm}$ to $22 \mathrm{~cm}$ for the range of plasma densities being considered. A low voltage sheath can be estimated as being a few Debye lengths thick, while a high voltage sheath can be tens of Debye lengths thick. This dictates a sheath size ranging from about $1 \mathrm{~cm}$ to tens of centimeters. (Lieberman \& Lichtenberg, 1994) These parameters, and the parameters measured in chamber tests as mentioned below are used in the PIC simulations.

\section{Vacuum Chamber Tests}

FEAC tests will be performed in the Cathode Test Facility (CTF) at the Plasmadynamics and Electric Propulsion Laboratory at the University of Michigan.

\section{Cathode Test Facility}

The CTF is a two meters long by 60 centimeters in diameter chamber that is pumped by a 135 CFM mechanical pump for roughing and a CVI TM500 (20 inch) cryopump with a measured xenon pumping speed of 1,500 1/s. Base pressure for the facility is $2 \times 10-8$ Torr. The facility consists largely of components either granted to PEPL by NASA Lewis Research Center or bought with funds from NASA Lewis Research Center and the Jet Propulsion Laboratory.

\section{Test Plan}

Under NASA sponsorship, environmental tests of sample FEACs will be performed. These devices will be subjected to a variety of neutral gas densities and constituents. Operational and survival limits will be determined for molybdenum, $\mathrm{ZrC}$ coated molybdenum, and other tips.

Space charge limit tests will also be performed via a variable plasma density, and mobile anode (current collector). Xenon plasma will be generated with a hollow cathode assembly. The ability to emit current across a limited gap will be compared to PIC simulation results, and the ability of a FEAC to emit charge into a plasma with no collector will be confirmed.

The test procedure will be automated insofar as possible, with GPIB devices and a Labviews controller. Current and voltage measurements of the gate, tips, collector, and spacecraft simulator (a steel can) will be made. The bias of the various components will be varied, thus experimenting with the various electrical configurations as described above, and with the effect of sheath size. Plasma density measurements in proximity to the emitter will be made with multiple Langmuir probes. Neutral pressure will be monitored with an ion gauge and residual gas analyzer. 


\title{
SUMMARY
}

FEAC systems are an enabling technology for EDT propulsion. PIC analysis and chamber tests are being performed to characterize and mitigate the various technical issues facing FEAC systems in the ionospheric region. This research will lead to efficient and effective designs which will make possible a variety of EDT and other missions.

\section{NOMENCLATURE}

\author{
$\varepsilon_{0}:$ permittivity of free space \\ e: electron charge $(\mathrm{C})$ \\ $\mathrm{m}_{\mathrm{e}}$ : electron mass $(\mathrm{kg})$ \\ $\mathrm{T}_{\mathrm{o}}$ : electron emission energy $(\mathrm{eV})$ \\ D: gap spacing $(\mathrm{m})$ \\ $\mathrm{V}$ : gap voltage (V) equal to spacecraft bias with respect to the plasma \\ W: emitter width \\ $\mathrm{r}_{\mathrm{b}}$ : emitter radius \\ A: emitter area $\left(\mathrm{m}^{2}\right)$ \\ $\mathrm{s}=$ sheath size $(\mathrm{m})$ \\ $\mathrm{I}_{\mathrm{CL}}(\mathrm{N})=\mathrm{N}$ dimensional Child-Langmuir current limit $(\mathrm{A})$
}

\section{ACKNOWLEDGMENT}

This work is being funded by a NASA-MSFC GSRP student fellowship and grant.

\section{REFERENCES}

Humphries, S., Charged Particle Beams, John Wiley and Sons, Inc., 1990.

Lieberman, Lichtenberg, Principles of Plasma Discharges and Materials Processing, John Wiley \& Sons, Inc., 1994

Luginsland, J., and McGee, S., and Lau, Y. Y., "Virtual cathode formation due to electromagnetic transients", IEEE Trans. Plasma Sci., 26, pp. 901-904, (1998).

Luginsland, J. W., and Lau, Y. Y., and Gilgenbach, R. M., "Two-dimensional Child-Langmuir law", Phys. Rev. Lett., 77, p. 4668, (1996).

Mackie, W. A., Xie, T., et al., "Emission and processing requirements for carbide films on Mo field emitters", Materials Issues in Vacuum Microelectronics Vol. 509, edited by Zhu, Pan, Felter, Holland; MRS, Warrendale, PA, 1998, p173-178

Marrese, C., Compatibility of field emission cathode and electric propulsion technologies: theoretical and experimental performance evaluations and cathode requirements, Ph.D., University of Michigan, 1999.

NRL, Plasma Formulary, published by the Naval Research Laboratory, 1998

Takemura H., Tomihari Y., et al., "A novel vertical current limiter fabricated with a deep trench forming technology for highly reliable field emitter arrays", Tech. Dig. IEEE-IEDM, p709-712; (1997)

Tascione, Thomas F., Introduction to the Space Environment, Orbit Book Company, Malabar Florida, 1988

Verboncoeur, J. P., and Alzes, M. V., and Vahedi, V., and Birdsall, C., "Simultaneous Potential and Circuit Solution for 1-D Bounded Plasma Particle Simulator Codes", J. Comp. Phys., V. 104, p321-328, (1993) 